\title{
Storia delle storie di Lanval
}

È noto che nell'universo della narrativa mondiale, ben poco c'è davvero di nuovo sotto il sole. Gli studiosi di novellistica, negli anni a cavallo dei due ultimi secoli, hanno rintracciato, con una erudizione che oggi lascia stupefatti, gli ascendenti dei motivi delle storie medievali, inseguendoli di secolo in secolo e di popolazione in popolazione dai paesi dell'Europa occidentale a quelli impregnati di cultura araba fino alle lontane contrade dell'India. Una parte della critica moderna, d'altra parte, basandosi sulla accertata ricorrenza dei motivi e dei patterns narrativi, tenta di identificare le leggi universali della narrativa e/o i loro presupposti psicologici, approdando a volte ad accostamenti apparentemente incongrui (come la storia biblica di Adamo ed Eva e la moderna detective-story, per esempio).

Pur utilizzando in parte dati già acquisiti per merito della critica storica a cui si accennava, scopo dello studio di cui si presentano qui i risultati è piuttosto di mettere in rilievo in una prospettiva rigorosamente storico-letteraria come, in uno spazio geografico e temporale delimitato, un determinato motivo narrativo si è conservato e come si è trasformato nel suo "incarnarsi" in generi letterari diversi; generi letterari a loro volta collegati a un'epoca, a una società, a una mentalità di volta in volta differenti.

Piú che di un motivo narrativo, in realtà si tratta di un intreccio di motivi concatenati in un racconto coerente, dunque una storia; e lo spazio geografico e temporale sono la Francia e l'Italia dal $\mathrm{XII}^{\circ}$ alla fine del $\mathrm{XIV}^{\circ}$ secolo.

\section{Il "lai de Lanval"}

$\mathrm{Al}$ centro del primo periodo di questa vicenda è il lai Lanval, uno della raccolta di Marie de France che, negli anni sessanta del $\mathrm{XII}^{\circ}$ secolo ha accolto il genere del lai narrativo per rilanciarlo a piú alta fortuna. Per quanto non si abbiano notizie sicure sulla sua vita, Marie de France sembra aver vissuto nell'entourage della corte 
normanna di Enrico II; i suoi Lais sono fra le creazioni poetiche piú raffinate e piú alte del $\mathrm{XII}^{\circ}$ secolo. È anche uno dei primi scrittori ad accogliere nelle sue composizioni motivi e fabulae derivati non piú dal patrimonio classico o storico ma dal patrimonio narrativo celtico. Nella nostra storia, il lai Lanval si pone non soltanto come punto di partenza di tutta l'elaborazione successiva ma anche, in un certo senso, come punto d'arrivo di un travaglio narrativo precedente. $\mathrm{E}$, in effetti, T.P. Cross, sezionando la storia del Lanval in una serie di figure e di motivi, ha rintracciato per ciascuno di essi gli antecedenti esistenti, prima della metà del $\mathrm{XII}^{\circ}$ secolo, nelle narrazioni celtiche. Cross tendeva a dimostrare che "the Lays of Lanval and Graelent are ultimately based on Celtic tales in which the fée seeks out her lover in the land of mortals, becomes his mistress, and lays upon him commands, the breach of which results in the severance of their relations"; ${ }^{1}$ e la sua ricerca sembra tesa a provare la probabilità che una storia come quella narrata nei lais appartenesse già al patrimonio celtico. La mia interpretazione è piuttosto che Maria abbia intrecciato, fino a farne un racconto coerente, motivi che nella narrativa celtica si trovano piú o meno allo stato sparso e integrati in narrazioni diverse. Ma precisiamo. Cross indica un accostamento assai preciso fra l'intreccio del Lanval e quello della storia Aidead Muirchertaig maic Erca (La morte di M. figlio di E.): in entrambe le narrazioni una donna soprannaturale si presenta al suo amato dichiarandogli che per amor suo è venuta a incontrarlo, ma gli impone una condizione (di non pronunciare mai il suo nome, Sín, nel racconto celtico, di non parlare della loro relazione nel Lanval), e ne segue un periodo di amore e di prosperità per il fortunato mortale; in entrambe le narrazioni, il tabú viene infranto e le conseguenze sono terribili per il mortale, poiché è in questa circostanza che il re Nuirchertaig muore, e Lanval si trova molto vicino a fare la stessa fine.

Anche nel Togail Bruidne Dá Derga troviamo un incontro d'amore fra il re Eochaid Feidleche e la fata Etain; e qui, come nel Lanval, siamo in presenza di uno specchio d'acqua, e la fata si sta lavando in un bacino d'argento (il bagno tornerà in un lai successivo al Lanval, il Graelent). Come nel Lanval si ha una descrizione abbagliata e particolareggiata dell'aspetto fisico della donna; seguono, come nel Muirchertaig, la dichiarazione che essa è venuta in quel luogo per amor suo, l'innamoramento subitaneo del re e il matrimonio. 
Per le conseguenze a cui va incontro Lanval dopo aver tradito il segreto della fata, il Lanval si avvicina piuttosto a due altri racconti: il Noínden Ulad (La debolezza novenaria degli uomini dell'Ulster) $^{2}$ e la Storia di Taliesin. ${ }^{3}$ Nel Noínden, il ricco contadino Crunniuc, la cui moglie soprannaturale lo ha ammonito a non vantarsi, si reca un giorno a una grande festa degli Ultonians. In quell'occasione i cavalli del re vincono una corsa e tutto il popolo proclama che questi cavalli sono decisamente i piú veloci di tutta l'Irlanda. Ma Crunniuc esce fuori con l'affermazione: "Mia moglie corre piú veloce di questi cavalli." ${ }^{4}$ Il re ordina allora di catturare Crunniuc e di tenerlo prigioniero finché la moglie non avrà fatto una gara di corsa con i suoi cavalli. La moglie, confermando che l'incauta vanteria del marito ha posto fine alla loro relazione, si reca a corte e, nonostante si trovi in stato di avanzata gravidanza, si impegna nella corsa con i cavalli, li vince, dà alla luce due gemelli e muore, lanciando però una maledizione sugli Ultonians: che periodicamente, fino alla nona generazione gli Ultonians siano presi da una debolezza simile a quella di una donna nel travaglio del parto.

Nella Storia di Taliesin è questione del giovane nobile Elphin, di sua moglie e del suo bardo Taliesin. Cito da una traduzione inglese: ${ }^{5}$

From that moment on, Elphin's wealth increased more and more each succeeding day, as well as his favor and acceptance with the king. Some while after this, at the feast of Christmas, the king was holding open court at Deganwy Castle, and all his lords - both spiritual and temporal - were there, with a multitude of knights and squires. Their conversation grew, as they queried one another, saying: "Is there in the entire world a man as powerful as Maelgwn the king? Or one to whom the heavenly Father has given as many spiritual gifts as God has given him: beauty, shape, nobility, and strength, besides all the powers of the soul?" And with these gifts, they proclaimed that the Father had given him an excellent gift, one that surpassed all of the others, namely, the beauty, appearance, demeanor, wisdom and faithfulness of his queen. In these virtues, she excelled all the ladies and daughters of the nobility in the entire land. Beside that, they asked themselves: "whose men are more valiant? Whose horses and hounds are swifter and fairer? Whose bards more proficient and wiser than Maelgwn's?"

And so after everyone had spoken in praise of the king and his blessings, Elphin happened to say this: "Indeed, no one can compete with a king except another king; but, truly, were he not a king, I would surely say that I have a wife as chaste as any lady in the kingdom. Furthermore, I have a bard who is more proficient than all the king's bards." 
Some time later, the king's companions told him the extent of Elphin's boast, and the king commanded that he be put into a secure prison until he could get confirmation of his wife's chastity and his poet's knowledge.

Come si vede, si ritrovano nel Noinden Ulad il vanto di possedere qualcosa di migliore del re, che causa l'indignazione del re e l'ingiunzione di provare il vanto, la posizione sotto processo, la rottura della relazione a causa del tabú infranto, il salvataggio da parte della donna che si presta a far verificare il vanto dell'amato, che sono i nodi caratteristici della trama del Lanval; mentre un diretto confronto fra la moglie del re e la donna del cavaliere si istituisce anche nella Storia di Taliesin.

Ma Maria raccoglie i motivi celtici espungendone tutti gli elementi che potevano apparirle barbarici, e inserendoli in un contesto che utilizza gli apporti delle opere letterarie cortesi cronologicamente vicine. Cosí, colloca la vicenda alla corte del re Artú, probabilmente ricordandosi del Brut di Wace, modifica l'incontro sulle rive di uno specchio d'acqua per adottare una sontuosa descrizione della tenda, sotto cui giace la fata, derivata dal Roman de Thèbes; la vanteria del cavaliere parendole gratuita e ingiustificata, inserisce il motivo del tentativo di seduzione da parte della regina, con un'accusa di sodomia che ricorda l'analoga accusa lanciata dalla madre di Lavinia ai Troiani nel Roman d'Enéas; dal giudizio di Gano della Chanson de Roland deriverebbe, secondo lo Hoepffner, la scena del giudizio di Lanval, e la triplice apparizione delle damigelle e della donna soprannaturale, alla fine della vicenda, ricorda lo stupore crescente di Kaherdin di fronte prima alle damigelle, poi alla regina Isotta, del Tristan di Thomas.

Insomma, sulla trama di un tema di fondo, quello dell'Offended Fée, presente nella letteratura celtica (come nella letteratura mitologica del mondo intero), e attingendo tranquillamente al repertorio di motivi delle narrazioni, orali e scritte, a lei contemporanee, Maria ha creato una storia che si pone come capostipite di una serie di rifacimenti o, ancor meglio, come ispiratrice di una serie di storie che, mantenendo del lai de Lanval alcune caratteristiche fondamentali, si presentano tuttavia sotto forme e con spiriti anche profondamente diversi.

E, prima di passare a studiare alcuni esemplari di questa serie originata dall'immensa fortuna dei lais di Maria, vorrei far già notare un passaggio di qualità, o piuttosto di genere, fra le narrazioni celtiche tramandateci e i lais. Gli studiosi hanno già rilevato il carattere "barbarico," "primitivo" dei costumi riflessi nelle nar- 
razioni celtiche, che le collega a un tipo di società profondamente diverso da quello della corte anglonormanna; ma sul piano narratologico è interessante osservare anche che, nelle narrazioni celtiche, spesso il racconto sbocca su una realtà che ne sta al di fuori: il racconto è cioè un'interpretazione o una spiegazione di un dato di fatto ritenuto appartenente all'ordine della realtà storica e non a quello dell'immaginazione. Cosí nel Noínden Ulad si narra l'antecedente che dà ragione della debolezza dei guerrieri irlandesi; la storia della relazione di Muirchertaig con la dea Sín è la spiegazione di "come morí Muirchertaig": morte che è il dato di fatto, lo sbocco necessario che origina il racconto; e la Storia di Taliesin è orientata verso la presentazione di una serie di poesie del famoso bardo, ritenuto personaggio storico. I racconti celtici conservano cioè in parte il carattere del mito eziologico. Nel lai, l'unico sbocco sulla realtà è il lai musicale che era presumibilmente suonato e cantato dopo il racconto dell' "aventure" che ne era stata all'origine; ma, come nota Payen nel suo studio sulla struttura del lai, la natura del lai è anzitutto quella di essere un racconto. ${ }^{6}$ Ha una struttura narrativa precisa: "le lai me semble être avant tout l'histoire d'une certaine crise. Au début du poème, le protagoniste est présenté dans une situation de manque. . . . Vient le moment où le héros connaît . . . le bonheur; mais ce bonheur est précaire et débouche sur l'épreuve. Un troisième moment est donc la description de cette épreuve. Le dénouement, heureux ou douloureux, est toujours abrupt. ${ }^{7} \mathrm{E}$, per di piú, è un racconto dichiaratamente fantastico, dunque d'immaginazione e non realistico. Secondo Frappier, la "crisi," in senso narrativo, del lai è determinata generalmente dall'irruzione nel nostro comune mondo dei mortali di elementi che appartengono ad un mondo altro, il mondo degli dèi o dell'aldilà: è ciò che determina l'"aventure." Naturalmente, il privilegio di entrare in contatto con questo mondo soprannaturale non può toccare che a un essere d'eccezione, un predestinato, che solo può riuscire a superare la prova indotta da questa rottura dell'ordine naturale delle cose (e anche quando il soprannaturale è assente dal lai, l'"aventure" resta qualcosa di eccezionale, di meraviglioso, di extra-ordinario). Queste definizioni si applicano, evidentemente, non soltanto ai lais di Marie de France ma al lai in quanto genere letterario: sembra effettivamente che esistessero lais narrativi piú antichi di quelli di Marie de France; ${ }^{8}$ ne esistono certo parecchi piú tardi. In nessun altro si fa allusione al lai musicale di cui l' "aventure" narrata da Marie de France sarebbe all'origine. Con il lai narrativo siamo 
dunque decisamente nell'ambito letterario - abbandonato ogni legame formale o contestuale con il mito.

\section{Il "lai de Graelent"9}

Un altro lai, anonimo, ha rielaborato, immediatamente dopo la sua apparizione come lai di Marie de France, la storia di Lanval. È nota l'incertezza che ha accompagnato i filologi nel descrivere i rapporti fra il Graelent e il Lanval. Rispetto al Lanval di Marie de France, il Graelent sposta all'inizio della storia il motivo della seduzione della regina, trasforma la scena dell'incontro con la fata contaminandola con il motivo della donna-cigno e, alla serena e gloriosa affermazione della dea che dichiara senza ambagi il suo amore a Lanval sostituisce la violenza carnale del cavaliere nei confronti della donna indifesa; l'occasione in cui Graelent tradisce il segreto del suo amore soprannaturale è l'esibizione, da parte del re, della regina perché tutti l'ammirino e affermino che è la piú bella di tutte le donne. Infine, nell'epilogo della storia, la fata parte da corte ancora sdegnata contro Graelent ed è necessario che l'eroe, correndole dietro, si slanci per ben due volte nell'acqua perigliosa di una "rivière infranchissable," rischiando la vita disperatamente, perché la fata si lasci commuovere dalle preghiere delle sue damigelle e conduca con sé l'eroe. Il suo cavallo, rimasto indietro sulla riva (dalla parte del nostro mondo), fu sentito ancora per lungo tempo nitrire lamentando la separazione dal suo padrone, e nessuno poté mai piú catturarlo.

Nonostante la goffaggine di alcune delle sue soluzioni narrative, il lai de Graelent ha affascinato gli studiosi per la suggestività di alcuni suoi motivi che sembrano rivelare una piú stretta affinità con motivi analoghi dei racconti celtici: torna in mente che anche il re Eochaid vede la fata Etain mentre si bagna presso una fonte, e che la fa catturare dai suoi uomini prima di parlarle; la lode pubblica di qualcosa che appartiene al re, contraddetta soltanto dall'eroe, ricorda da vicino l'episodio corrispondente del Noínden Ulad e, ancor piú, la Storia di Taliesin; la "rivière infranchissable" è un luogo comune della mitologia celtica per indicare il confine fra il nostro mondo e quello dell'aldilà; il nitrito disperato del cavallo che ancora si sente echeggiare ricorda analoghi esempi di cavalli che, quasi umanizzati, lamentano le sorti del padrone. Si è affermato quindi abbastanza spesso che il Graelent e il Lanval sono rielaborazioni distinte di una medesima narrazione perduta, antecedente naturalmente ai due lais. Piú recentemente, Cesare Segre (riprendendo osservazioni di Foulet et Hoepffner) ha dimostrato 
in maniera ineccepibile che il Graelent ha riutilizzato, nella sua stesura, versi dei Lais di Marie de France, presi non soltanto dal Lanval ma anche da altri (Eliduc, Guigemar). Il Graelent non può essere dunque che posteriore a Marie de France e non può essere che una rielaborazione del Lanval. Segre sorvola però un po' rapidamente sulla questione dei motivi che, nel Graelent, coincidono, come abbiamo visto, con narrazioni piú antiche di Marie de France. A me pare che quest'apparente contraddizione possa essere spiegata col fatto che l'autore del Graelent, pur riproducendo fondamentalmente la storia che nelle sue articolazioni e nella sua coerenza è stata creata da Marie de France, ha però riconosciuto il patrimonio folkloristico a cui Marie aveva attinto e, una volta richiamati alla memoria i motivi celtici di cui Marie aveva intessuto il suo racconto, ha attinto a sua volta allo stesso patrimonio, introducendo elementi degli stessi episodi che Marie non aveva raccolto, o riproducendo altri motivi ancora.

In ogni modo, è certo che il Graelent dev'essere stato composto a breve distanza di tempo dal Lanval di Marie e che dové godere di una notorietà e di una diffusione altrettanto, se non piú grande. $\grave{E}$ un fatto che tanto il lai inglese Sir Launfal, ${ }^{10}$ quanto il poema Partonopeus de Blois, quanto il cantare italiano del Bel Gherardino utilizzano elementi del Graelent, accanto a quelli del Lanval, e Graelent si trova spesso menzionato, come personaggio o come storia di successo, in fonti anteriori alla fine del XIII ${ }^{\circ}$ secolo. ${ }^{11}$

\section{Il "Partonopeus de Blois"}

Il Partonopeus non è stato datato con precisione, ma è collocato dalla maggior parte degli studiosi intorno al $1180 .{ }^{12}$ Qui assistiamo ad uno spostamento di un certo rilievo nella rielaborazione della storia di Lanval, che è trasformata in un vero e proprio poema, lungo dai 9,738 ai 14,493 versi, $^{13}$ a seconda delle versioni e delle varie continuazioni. Rimangono, del Lanval, le linee essenziali: un giovane cavaliere è condotto presso una donna dai poteri soprannaturali e ne è colmato di amore e di ricchezze; la donna gli impone però una proibizione che il cavaliere, tornato alla sua corte d'origine, infrange, provocando la rottura della relazione e lo sdegno della donna. Il cavaliere cade in una profonda disperazione, che lo conduce presso alla morte. Diversi tratti del poema svelano l'ascendenza di altri lais imparentati col Lanval: Partonopeus è nipote del re e si perde durante una caccia come nel Guingamor (ma la caccia è già nel Graelent); la conquista della donna implica una violenza, il cavallo dell'eroe nitrisce lungo la riva di 
un'acqua, c'è un'opera di intercessione presso la donna sdegnata da parte di altre donne, come nel Graelent; l'eroe compie prodezze guerriere, con l'incoraggiamento della sua donna, dopo il primo incontro, teme che la sua donna sia "di mala natura" ed è rassicurato su questo punto dalle pratiche religiose dell'amata, tradisce il segreto (o determina di infrangere la proibizione) durante l'incontro con un ecclesiastico, esce dalla camera della donna in una situazione di pericolo, scortato da una damigella, compare una sorella della donna amata come nel lai di Désiré. ${ }^{14}$

Un'innovazione fondamentale, che appartiene probabilmente in proprio all'autore del Partonopeus consiste nell'umanizzazione della vicenda: la donna dai poteri soprannaturali non è piú un essere divino o fatato, ma è la principessa di un regno ben umano, e i suoi poteri le sono stati dati dall'educazione ricevuta, particolarmente nelle arti magiche (non si dimentichi che nel medioevo la magia è una scienza ben umana). Ma l'innovazione che, alla luce delle rielaborazioni italiane successive acquista il maggiore rilievo avviene nella natura del tabú: al silenzio sulla loro relazione ingiunta dalla fata a Lanval, il poema sostituisce un motivo derivato dalla apuleiana favola di Amore e Psiche: ${ }^{15}$ i due giovani si incontrano cosí per la prima volta al buio, in un letto, sotto le stesse lenzuola. La principessa impone a Partonopeu di non tentare di vederla, per due anni, e contentarsi dei loro incontri notturni; l'infrazione consiste nello spiare le forme della donna con l'aiuto di una lampada. Viene cosí a cadere il motivo del vanto e del paragone di bellezza con la regina, e quindi la posizione sotto processo del cavaliere. Corrispondentemente, viene esaltato il motivo dello sdegno della donna e del pentimento del cavaliere - tratti derivati probabilmente dall'Yvain di Chrétien de Troyes. La soluzione dell'impasse viene non da un'epifania della donna ma dalla vittoria in un torneo indetto per scegliere lo sposo della principessa, che trova la sua ascendenza nei poemi di Ipomedon e di Cligès. ${ }^{16}$ Un altro incidente deriva da un episodio dello Chevalier à la charrette: l'eroe riesce a prender parte al torneo nonostante si trovi, in quel momento, prigioniero di un tiranno (che partecipa anche lui al torneo) perché la moglie del tiranno gli concede provvisoriamente la libertà e gli fornisce armi e cavallo. Ucciso il tiranno nel corso del torneo, l'eroe torna, fedele alla parola data, in prigione, ma ottiene dalla donna la libertà definitiva.

L'autore del Partonopeus ha dunque arricchito la trama di base, derivata dalla "storia di Lanval" con ogni possibile aggiunta e arricchimento proveniente dalle sue vaste conoscenze di materia 
classica e romanzesca, di modo che la natura stessa della struttura narrativa risulta fondamentalmente diversa. L'opposizione teorizzata da Zumthor fra struttura narrativa a "durée ouverte" del modèle romanesque 17 e struttura narrativa a "durée close" della nouvelle $^{18}$ potrebbe trovare una verifica appropriata in questa trattazione della stessa fabula nei due generi opposti del lai e del poema romanzesco. Si pensi alla concentrata conclusione del lai di Marie e invece, d'altra parte, al fatto che il poema del Partonopeus, al di là della conclusione della fabula con il matrimonio di Partonopeu e della principessa, presenta ancora ben due continuazioni d'autore: il ritrovamento di Anselot, con il lungo racconto delle sue vicende e l'infelice fine della sua storia d'amore, e la guerra condotta contro Partonopeu da uno dei contendenti alla mano della principessa, rimasto battuto nel giudizio finale.

Ma, sul piano dei documenti storici e non degli astratti modelli, abbiamo assistito piuttosto ad un progressivo arricchimento e allungamento della storia di Lanval particolarmente nel fargli raggiungere l'happy end - quasi che l'improvvisa risoluzione del conflitto fra i due amanti con il gesto di Lanval che si slancia sul cavallo stesso della donna mentre questa abbandona la corte, non soddisfacesse le esigenze narrative dei rifacitori, che avevano bisogno di dimostrare come l'eroe riacquistasse l'amore della donna per proprio merito, e non semplicemente per la generosa degnazione della fata. Ed ecco Graelent sfidare per ben due volte la morte nel tentativo di raggiungere la donna, ecco il vano tentativo di Désiré di raggiungere il figlio e la pericolosa incursione notturna nella camera della donna, ecco, soprattutto, l'introduzione del motivo del torneo nel Partonopeus.

Un secondo romanzo in versi, francese, Le Bel Inconnu, è indebitato evidentemente verso il Lanval e anche, a quanto sembra, al Partonopeus, ${ }^{19}$ ma senza conservarne la trama di fondo. Anzi, la "storia di Lanval" sembra essere utilizzata soprattutto per complicare le vicende della storia primaria, che appartiene a un altro filone, quello rappresentato separatamente in Italia dal cantare del Carduino. ${ }^{20} \mathrm{Ne}$ parleremo piú distesamente quando additeremo le sue relazioni con il cantare italiano del Bel Gherardino.

\section{La "Chastelaine de Vergi"}

Prima di lasciare il territorio francese per inseguire le continuazioni dei lais e dei poemi in ambiente italiano, converrà accennare ad ancora un altro documento, derivato dal lai di Marie, che per 
suo proprio conto fa da capostipite ad un'altra serie di continuazioni, anche italiane: il poemetto (948 vv.) della Châtelaine de Vergi.

La Châtelaine conserva, della storia originaria, la proibizione della donna al cavaliere di svelare la loro relazione, pena la rottura della relazione stessa (Lanval: "Ami, fet ele, or vus chasti, / Si vus comant e si vus pri: / Ne vous descouvrez a nul homme. . . . A tuz jurs m'avriez perdue, / si cest amur esteit seüe: / Ja mes ne me purriez veeir / Ne de mun cors seisine aveir. Vv. 143-50; CV: "la dame li otroia / Par itel couvenant s'amor, / Qu'il seüst qu'a l'eure et au jor / Que par soi serait descouverte / Leur amors, qu'il i auroit perte / et de l'amour et de l'otroi /Qu'ele li avoit fait de soi." Vv. 22-28), il tentativo di seduzione del cavaliere da parte della sovrana e la conseguente situazione di disgrazia e di condanna da parte del signore in cui il cavaliere viene a trovarsi. Anche la condanna è simile: se non proverà la verità del suo vanto, Lanval "Tut sun service pert del rei, / E sil dei cungeer de sei" (vv. 459-60); l'amante della châtelaine si sente ingiungere: "Issies errant hors de ma terre, / Que je vos en congié sans dote. / Et la vous vé et desfent toute" (vv. 170-72).

Come nel Lanval, e a differenza del Graelent, è l'episodio della seduzione che conduce necessariamente alla rivelazione del segreto; le conseguenze di questa infrazione nella storia della Châtelaine si allontanano invece notevolmente da quelle della storia di Lanval, poiché il fallo del cavaliere conduce entrambi gli amanti al suicidio. Inoltre, sul piano dello studio dei motivi, è interessante osservare che la Châtelaine risolve brillantemente un'aporia presente nel Lanval e causata dalla contaminazione, operata da Marie, fra due motivi contrastanti. Marie ha combinato, infatti, nel suo racconto, il motivo della lode alla moglie del re, la cui contraddizione costituisce un reato per scagionarsi del quale il cavaliere deve provare la verità del suo vanto, e il motivo del tentativo infruttuoso di seduzione da parte della sovrana che porta alla calunnia del cavaliere. Nel Lanval, stranamente, il re Artú e la regina danno piú importanza al vanto di avere un'amante piú bella che al supposto tentativo di seduzione:

Li reis . . .

As chambres la reine entra.

Quant el le vit, si se clamma,

As piez li chiet, merci li crie

Et dit que Lanval l'ad hunie:

De drüerie la requist;

Pur ceo qu'ele l'en escondist 
Mut la laidi e avila;
De tele amie se vanta
Qui tant iert cuinte e noble e fiere
Que meuz valut sa chamberere,
La plus povre, qui tant serveit,
Que la reine ne feseit. (Vv. 311-24)

Bisogna ammettere che c'è qui una certa incongruenza: se Lanval aveva un'amante cosí soddisfacente, perché avrebbe tentato di sedurre la regina? E il tentativo di seduzione stesso, non avrebbe dovuto costituire un'offesa ben piú grave del vanto di avere una donna tanto bella? E il duca della Châtelaine, infatti, minaccia di condannare il suo vassallo a causa dell'offesa portata al suo onore di marito e di signore; per poter continuare a seguire la sua fonte e introdurre nel racconto la necessità di rivelare l'identità dell'amata, l'autore della Châtelaine fa ricorso alle convenzioni della dottrina d'amore elaborata appunto in quel torno di tempo nelle corti francesi - e può farlo senza forzature perché in fondo è di queste che tutto il racconto è impregnato: il comportamento del cavaliere dimostra che è innamorato; siccome non si sa di chi, è bene ch'egli lo dica, altrimenti il duca crederà ch'egli ama sua moglie.

In effetti, piú che nel diverso svolgimento della storia, la grande differenza della Châtelaine de Vergi dal lai ispiratore consiste nella completa umanizzazione della vicenda, e nell'aver fatto di questa vicenda una storia esemplare delle convenzioni dell'amor cortese: la donna è una nipote del duca, e il segreto è imposto in quanto è una delle condizioni della fin'amor; conseguentemente, l'infrazione alle leggi dell'amor cortese non può che condurre a una fine tragica (all'opposto che nella storia di Lanval).

Anche qui si ha un chiaro esempio di un motivo ispiratore, anzi di una fonte vera e propria che, trasferita in un contesto diverso, e utilizzata ai fini di una significazione originale, è costretta ad essere profondamente modificata proprio per rispondere alla nuova natura del testo in cui è stata incorporata.

La Châtelaine è all'origine di un diverso filone letterario che, abbandonato decisamente ogni elemento fantastico, mette risolutamente in scena una vicenda che potrebbe essere contemporanea, con personaggi e situazioni chiaramente riconoscibili come facenti parte della realtà - vera o idealizzata - a cui appartengono narratore e ascoltatori. Non a caso potrà essere ripresa, senza trasformazioni profonde, dai novellieri francesi e italiani dei secoli seguenti, dopo aver fornito l'argomento al cantare della Dama del Verzú: "La Châtelaine de Vergi . . . n'est plus un lai et elle n'est pas 
encore une nouvelle. Oeuvre mêlée, elle marque en quelque sorte une transition, dans la mesure surtout où elle traduit, de la part de son auteur, un désir de renouvellement et une volonté de trouver une voie nouvelle."21

\section{Fase italiana}

Nel corso del XIII ${ }^{\circ}$ secolo il Partonopeus de Blois fu tradotto in tedesco, in neerlandese e in norvegese (dalla traduzione norvegese deriveranno una saga islandese e una versione danese). Due versioni inglesi appartengono al $\mathrm{XV}^{\circ}$ secolo; esistono inoltre una versione in prosa spagnola e una catalana, tardive. ${ }^{22}$ Per l'Italia, non si ha invece conoscenza di una vera e propria traduzione. Certo, il cantare del Bel Gherardino è considerato la versione italiana del Partonopeus de Blois, e vi sono, fra il poema e il cantare, abbastanza punti di contatto per iscrivere quest'ultimo nel filone delle derivazioni dalla "storia di Lanval"; ma, come vedremo, i rapporti fra il cantare e il poema sono un po' piú complicati. ${ }^{23}$

Piuttosto, l'influenza del Partonopeus si è manifestata in Italia, a una data antecedente a quella del Bel Gherardino, su un poema famoso senza che nessuno l'abbia ancora, mi sembra, riconosciuta: intendo parlare del Teseida del Boccaccio.

Si ripete che la fonte del Teseida è sconosciuta: forse si potrebbe mettere in dubbio l'esistenza stessa di una "fonte" e concedere al giovane Boccaccio la stessa stima che si concede ad altri romanzieri, pur medievali, che hanno intessuto i loro poemi di motivi e di episodi tratti da varie fonti, e hanno saputo combinare fra loro questi motivi e questi episodi in un tutto - una storia - coerente che è di per sé cosa nuova: vedi Marie de France, vedi Chétien de Troyes. Ora, fra le tante letture che hanno suggerito motivi ed episodi inseriti nella trama del Teseida, c'è anche, senza dubbio, il poema di Partonopeus.

L'idea stessa della guerra contro le Amazzoni potrebbe essere stata suggerita da un passo della versione vulgata relativa al sultano che contende fino in fondo la principessa a Partonopeu:

Et li noveax a non Alés de Femenie;

D'Amazoine fu nez, d'une terre florie:

Nus males hom n'i maint, de dames est garnie;

La mamele destre a chascune arse et broïe

Por arc et por espee et por lance brunie

Dont deffendent lor terre et font la garentie.

Li soudans Margariz, qui ceste ost a fornie,

Seur eles mut en ost o grant chevalerie. 
Quant conquerre nes pot, sin fist la resortie.

Porquant n'i fu endart, quar il i ot amie:

Lor roine l'ama, sin ot la druerie,

Sin engendra Alés. . . (G 169 a $)^{24}$

È menzionata espressamente l'isola di Tenedos, che nel Partonopeus è l'isola in cui l'eroe cade prigioniero. L'esercito di Teseo, venuto per mare, sbarca vicino a un "bel castel vicino al mare / sopra una montagnetta," come l'esercito del Sultano sbarca presso il castello di Malbreon; una fase importante della guerra si svolge sotto le sue mura; la guerra termina in seguito all'invio, da parte dell'avversario, di un'elaboratissima lettera. La trasformazione di Arcita lontano da Emilia a causa del suo dolore ricorda l'abbattimento di Partonopeu nella foresta delle Ardenne nel periodo del suo pentimento; ${ }^{25}$ l'episodio in cui Palemone, evaso di prigione, sorprende in un bosco l'amico che si sta svegliando e, nascosto dietro gli alberi, ascolta il suo soliloquio d'amore, ricorda l'analoga sorpresa di Partonopeu che, alla ricerca di due cani da caccia smarriti nella foresta, sorprende e ascolta il vaneggiamento del vecchio compagno Anselot; l'enorme spazio accordato al torneo, indetto per trovare uno sposo a Emilia, è analogo alla lunghissima trattazione del torneo che vedrà Partonopeu vincitore e sposo della principessa.

Si dirà che ciascuno di questi motivi è, di per sé, un luogo comune e che avrebbe potuto essere reperito anche in un'altra fonte; ma il trovarli tutti insieme, tanto nel Partonopeus che nel Teseida, fa propendere per la soluzione piú semplice, che il Boccaccio cioè li abbia tratti dalla sua conoscenza del poema francese.

Nello stesso Teseida, sembra di poter cogliere anche l'eco di una conoscenza del Lanval: la descrizione della bellezza di Emilia, collocata strutturalmente alla fine del poema (il giorno del matrimonio), che percorre a cavallo le vie della città suscitando in chiunque la vede ammirazione e stupore, ricorda la parusia della fata di Lanval, presentatasi anch'essa a cavallo alla corte del re Artú:

Il 'ot al burc petit ne grant

Ne li vieillard ne li enfant

$\mathrm{Ki}$ ne l'alassent esgarder

Si cum il la veent errer

......................

Li jugeur ki la veeint

A grant merveille la teneient.

Il n'i ot un sul ki l'esgardast
I teatri, le vie, piazze e balconi per li quali essa andando gir dovea al tempio là dov'erano i baroni, tutt'eran piene; e ogni uom vi

[correa, femine e maschi, e vecchi con [carzoni per veder questa mirabile dea; 
De dreite joie ne s'eschaufast. la qual ciascuno oltre ogn'altra (Lanval, vv. 575-84)

[lodava,

e per lo ben di lei Giove pregava.

(Teseida, XII, 66)

La larghezza e la generosità di Arcita e Palemone, liberati da Teseo, nell'attesa del torneo, ricordano ugualmente la generosità di Lanval-Graelent dell'epoca felice in cui la fata li colmava del suo amore e delle sue ricchezze. Ma si tratta, in ogni caso, di motivi isolati: il Teseida non s'iscrive nel filone delle storie di Lanval.

\section{Il "Bel Gherardino"}

Vi si iscrive invece, di pieno diritto, il cantare del Bel Gherardino già menzionato. Qui ritroviamo la trama di base: un giovane (che nell'ambiente fiorentino della metà del Trecento non è piú cavaliere, ma ha il "cor gentile" dell'essere nobile) acquista, quasi per caso, l'amore di una Fata; questa gli regala un guanto che lo fornirà di tutte le ricchezze, ma gli ingiunge di non parlare di lei, quando tornerà al suo paese. La madre di Gherardino riesce a carpirgli il segreto e il giovane si ritrova povero come prima. Intraprende allora un viaggio di ritorno alla ricerca dell'amata, ma rischia di affogare, insieme a un suo fedele amico, attraversando un fiume; la sorella della Fata li salva e li conduce in una sua isola; Gherardino è trasportato da una tempesta in un altro regno e fatto prigioniero. La Fata Bianca intanto indice un torneo promettendo la sua mano al vincitore; Gherardino è liberato dalla moglie del Sultano che lo ha fatto prigioniero, partecipa al torneo, vince e riottiene la mano e l'amore della Fata; l'amico di Gherardino sposa la sorella della fata, la sultana rimasta vedova è data in sposa a un "nobile donzello."

Nella sua ultima parte il cantare coincide, evidentemente, con il poema del Partonopeus (nella versione del ms. A che, a differenza di tutti gli altri, si conclude con la morte del Sultano e con un triplice matrimonio). Concordano col poema, inoltre, il motivo dei servitori invisibili, l'incontro nel letto, i nuovi abiti che il giovane trova al suo risveglio, i diporti in cui passa le giornate in attesa di ritrovare la sua bella di notte, il ruolo affidato alla madre nell'indurre l'eroe a rinunciare al suo segreto. Tuttavia, la dama non è affatto la principessa di Costantinopoli ma torna ad essere una fata, sia pure castellana; la proibizione non è quella di vederla, ma torna ad essere quella di parlare di lei; il giovane non è un grande 
signore, cugino del re di Francia, ma torna ad essere un giovane povero a cui l'amore della fata porta contemporaneamente il favore della ricchezza e la gioia di poter donare largamente. Mancano inoltre totalmente l'intermezzo epico del primo ritorno di Partonopeu in patria, la seduzione della nipote del re, il raddoppio dell'allontanamento di Partonopeu dalla bella, la disperazione del pentimento che lo riduce a "uom selvaggio." Il Bel Gherardino ci rimanda cioè a uno stadio della storia anteriore a quello del Partonopeus.

Per render conto di queste discrepanze, già il Levi aveva affermato: "Probabilmente l'autore del cantare non aveva sott'occhio l'intero poema del Partonopeus ma un compendio in prosa o in verso, forse uno dei numerosissimi testi franco-veneti che sono andati perduti"; ma definiva comunque i rapporti fra il Gherardino e il poema francese in questo modo: "Il cantare segue dunque, nei suoi tratti essenziali, il poema francese, ma omette molte parti che sono caratteristiche e belle . . . e aggiunge il duplice bagno del bel Gherardino desumendolo dalla leggenda di Graelent." 26

Oltre ai lais anonimi, al poema del Partonopeus e al poemetto della Châtelaine de Vergi, altri poemetti francesi imitano il lai di Marie de France, pur modificandone i motivi e inserendoli in contesti diversi. Cosí Le Bel Inconnu, presumibilmente di Renaut de Beaujeu e scritto fra la fine del $\mathrm{XII}^{\circ}$ e l'inizio del XIII ${ }^{\circ}$ secolo, rivela, particolarmente nelle descrizioni delle varie "puceles" messe in scena, la suggestione esercitata dalle descrizioni della donna di Lanval; quanto alla trama, anche qui è questione di un cavaliere che, dopo varie avventure, incontra una castellana, la "Pucele as Blanches Mains" ("Onques nus hom ne vit si bele; / Le set ars sot et encanter / et sot bien esteles garder / Et bien et mal, tut ço savoit": vv. 1932-35), e ne guadagna l'amore, ma l'abbandona per adempiere a doveri cavallereschi; pentito e mortificato, ritorna presso la bella che dopo un periodo di prova lo riammette nei suoi favori.

Le Bel Inconnu è uno strano poema perché combina insieme due storie: quella che abbiamo appena delineata, e che vede come protagonista femminile una donna dai poteri soprannaturali, e quella che si trova ritratta in un altro cantare italiano, Carduino, del cavaliere che parte dalla corte di re Artú per andare a salvare una fanciulla imprigionata da un malvagio incantatore sotto le spoglie di un'orrenda biscia; e la liberazione avviene tramite il motivo del "fier baiser": il bacio della biscia al cavaliere. Il risultato di questa combinazione è alquanto incongruo perché il cavaliere, innamo- 
rato della fata e felicemente corrisposto, finisce invece per sposare, in seguito a un torneo di cui riesce vincitore, la fanciulla liberata dall'incanto; e la contraddizione è ben presente alla coscienza dell'autore che presenta la conclusione del poema a questo punto come un artificio per ottenere dalla sua propria dama un "bel sanblant":

Quant vos plaira, dira avant,

U il se taira ore a tant.

Mais por un biau sanblant mostrer

Vos feroit Guinglain retrover

S'amie, que il a perdue,

Qu'entre ses bras le tenroit nue.

Se de çou li faites delai,

$\mathrm{Si}$ ert Guinglains en tel esmai

Que ja mais n'avera s'amie.

D'autre vengeance n'a il mie,

Mais por la soie grant grevance

Ert sor Guinglain ceste vengance,

Que ja mais jor n'en parlerai

Tant que le bel sanblant avrai. (Vv. 6253-66)

Ora, il Bel Gherardino rivela vistose coincidenze con questo poema, ad esclusione del Partonopeus: l'incontro con la Fata castellana avviene dopo exploit cavallereschi, uno dei quali avviene proprio in vista del castello; ${ }^{27}$ di questo è sottolineata l'eccezionale fortezza e bellezza:

Il esgarde, voit un castiel,

Onques nus hom ne vit si biel.

De blanc mabre [sic] li mur [estoient,

Qui le castiel entor clooient,

$\mathrm{Si}$ hals con pooit $\mathrm{s}$ ars traire

Et tant estoient li mur halt

Qu'il ne doutoient nul assaut.

(Vv. 1875-96)

Un palais $\mathrm{i}$ ot bon et biel

(v. 1904)
E raguardando per quella pianura ebbe veduto uno nobile castello ch'era cerchiato d'altissime mura, ch'al mondo non aveva uno pari di [quello.

(I, 11, 1-4)

Ed entro si vvi aveva uno bello [palagio $(\mathrm{I}, 11,7)$

Il Bel Inconnu ha un fedele scudiero (Robers) che lo accompagna lungo tutta la storia, come il Marco Bello di Gherardino; la dama 
del poema francese si chiama la Pucele as Blanches Mains, ma l'autore ha l'occasione di sottolineare che la sua bianchezza andava ben oltre le mani:

Li sebelins, qui noirs estoit,

Les le blanc vis molt avenoit;

$N^{\prime}$ avoit vestu fors sa chemisse,

Qui plus estoit blance a devise

Que n'est la nois qui ciet sor branche.

Molt estoit la cemisse blance,

Mais encore est la cars molt plus

Que la cemisse de desus.

Les ganbes vit, blances estoient,

Qui un petit aparissoient;

La cemisse brunete estoit

Envers les janbes qu'il veoit. (Vv. 2403-14)

Ses mameles et sa poitrine

Furent blances con flors d'espine. (Vv. 2429-30), ${ }^{28}$

descrizione che giustifica pienamente l'appellativo del cantare italiano, la Fata Bianca. La scena d'amore fra il cavaliere e la fanciulla ha corrispondenze puntuali:

Ains le comenche a enbrachier

Entre ses bras molt doucement.

(Vv. 2380-81)

Son pis sor le sien il tenoit

$\mathrm{Nu}$ a nu. ... (Vv. 2435-36) e la donzella fra le braccia prese, che di bellezze non avea difetto, e sopra il bianco petto si distesse,

(I, 24, 3-6)

Portata a conclusione la vicenda dell'eroe con la riconciliazione con la Pucele as Blanches Mains, l'autore del Bel Inconnu passa subito all'episodio del torneo, e al conseguente matrimonio che conclude il romanzo (e, come nel Bel Inconnu, anche nel Bel Gherardino l'eroe, udita la notizia del torneo, si toglie dalle braccia di un'amante che lo lascia andare malvolentieri); questo può dar ragione del carattere compendioso con cui il Bel Gherardino si presenta rispetto al Partonopeus: anche nel B.G., infatti, la storia dell'Offended Fée si riconnette immediatamente all'ultima parte del Partonopeus, omettendo tutte quelle parti "caratteristiche e belle" la cui assenza era stata notata dal Levi.

Infine, non sarà sfuggito l'appellativo "bel" dell'eroe; ${ }^{29}$ il nome stesso, Gherardino, sembra avvicinarsi piuttosto a Graelent o a 
Guinglain (il vero nome del bel inconnu) che a Lanval o Portonopeu.

In conclusione, se non si può negare un'influenza diretta del Partonopeus sul Bel Gherardino, appare anche che il cantare, o il "libro" che l'autore metteva in rima, si rifaceva piuttosto ad un racconto, Li Biaus Desconeüs, o la sua fonte, che conservava legami con la forma primitiva della storia e, pur già influenzato dal Partonopeus, presentava innovazioni sue proprie, trasmessi, gli uni e le altre, al Bel Gherardino, e assenti dal Partonopeus.

Quanto alla particolarità del "genere" cantare, si è sorpresi in realtà di constatare come, a metà del $\mathrm{XIV}^{\circ}$ secolo, il cantare italiano conserva immutati certi caratteri dei poemetti francesi del secolo XII ${ }^{\circ}$ exeunte: priorità accordata all'intento narrativo, atteggiamento di ingenuo rapimento di fronte allo splendore delle cose e delle gesta descritte, forte presenza del meraviglioso; sul piano della forma, il couplet d'octosyllabes è sostituito dalla misura dell'ottava, che induce un découpage narrativo piú complesso, a quadri successivi; ${ }^{30}$ ma non si tratta che del raffinamento di una forma eminentemente narrativa.

È vero che Gherardino non è piú un cavaliere ma un giovane borghese che, per la sua eccessiva larghezza, si riduce in miseria ed è rinnegato dai fratelli - migliori massai: è scomparsa la sostanza feudale avvertibile nel Lanzal e nel Partonopeus, è scomparso anche l'indugio nelle descrizioni della bellezza e della ricchezza degli addobbi delle dame che pascevano la fantasia del cantore e del pubblico del Bel Inconnu (nei comuni italiani le manifestazioni eccessive di lusso erano severamente proibite!); ma si tratta, come si vede, di un adattamento superficiale all'ambiente cui è destinata la recitazione. In realtà, una vera e propria continuità di tono ricollega il cantare del $\mathrm{XIV}^{\circ}$ secolo ai poemi del $\mathrm{XII}^{\circ}$ e $\mathrm{XIII}^{\circ}$ secolo. ${ }^{31}$

Se una caratteristica si riesce a individuare nei cantari che ripetono la storia di Lanval, questa sarà da ricercare, piuttosto, al livello della configurazione della struttura narrativa: il racconto è diviso nettamente in due parti, la prima dedicata alle vicende che portano all'acquisto di un bene desiderabile, e alla sua perdita; la seconda dedicata alla quête che conduce necessariamente alla riconquista di questo bene: per la natura stessa della quête, tutti gli episodi presentati sono concatenati in modo da portare alla conclusione, che è contemporaneamente quella della storia e quella della narrazione. In alcuni cantari, la divisione strutturale ha una consacrazione anche formale nella divisione in due cantari; in altri 
lo svolgimento è continuo; ma già all'altezza del primo cantare a noi trasmesso, il Fiorio e Biancifiore, osserviamo che, mentre nel poemetto francese le premesse alla ricerca di Florio sono risolte in 513 versi su 3032, nel cantare la parte corrispondente occupa 63 ottave su 123. Una tale divisione della materia si è consolidata probabilmente perché rispondeva alle esigenze funzionali della recitazione pubblica "a puntate," ma è interessante anche perché corrisponde a una convenzione che si stabilisce fra pubblico e dicitore, in cui gli ascoltatori vengono resi consapevoli dei meccanismi di costruzione del racconto loro presentato. Alle stesse esigenze funzionali risponde anche l'esaltazione della dinamica del racconto (il mythos) a discapito di qualunque forma di indugio lirico (l'ethos), ancora presente nei poemetti francesi.

Il Bel Gherardino è il primo cantare a noi conservato che presenta la divisione formale in due cantari.

\section{Il "Liombruno." La "Ponzela Gaia"}

Il Liombruno e la Ponzela Gaia, trasmessi da manoscritti quattrocenteschi, conservano però molto piú fedelmente del Gherardino elementi caratteristici del lai di Marie de France, rendendo indiscutibile la presenza in Italia, nella seconda metà del Trecento, di questo lai vecchio di due secoli. Cosí, il Liombruno ripresenta il motivo del vanto dinanzi al re, la necessità - pena una severissima condanna - di provarlo e l'impossibilità di farlo, e l'apparizione della fata preceduta per ben due volte dalle sue "cameriere." Al contrario che nel Lanval, però, il ritorno della fata non significa perdono e riconciliazione, ma segna il punto piú profondo dell'infelicità dell'eroe:

Liombrun perdonanza gli chiedia.

Ed ella disse: - Falso rinnegato,

Della tua morte non m'incresceria. -

Per altra via la donna se n'andava,

Né arme né caval non gli lasciava. (Liombruno, vv. 372-76)

E qui comincia la quête di Liombruno, che si svolge per vie interamente diverse da quelle della tradizione della storia.

La Ponzela Gaia presenta un'ancor piú impressionante vicinanza al lai de Lanval: un cavaliere di Artú (Galvano) incontra nel bosco la figlia di Morgana, venuta alla sua ricerca perché innamorata di lui e ne riceve la solita proibizione; tornato a corte la regina tenta di sedurlo e, respinta, provoca una gara di vanti fra i cavalieri della 
corte. Poiché Galvano non vi partecipa, la regina lo rimprovera aspramente e lo induce a svelare il suo segreto; i vanti devono essere provati: Galvano non riesce, naturalmente, a provare il suo ed è condannato a morte finché l'apparizione della Ponzela Gaia non lo scagiona e non lo riammette nel favore della corte. La Ponzela Gaia sarà però punita dalla madre per il suo amore e incarcerata nel fondo di una torre, finché Galvano non verrà a liberarla.

Dunque, mentre il Bel Gherardino si spiega interamente con la conoscenza di rielaborazioni del lai di Marie de France (Partonopeus, Bel Inconnu, Graelent), gli altri due cantari hanno invece rintracciato e attinto direttamente alla fonte primitiva della storia, oltre che a fonti di vario genere, non connesse con la storia di Lanval; ma, d'altra parte, sembra possibile riconoscere un'influenza del Bel Gherardino sul Liombruno, e del Liombruno sulla Ponzela Gaia, che rende veramente interessante questo intreccio di prestiti fra testi appartenenti tutti al medesimo filone narrativo, e che può servire a stabilirne la cronologia, almeno relativa. E infatti. Nel Bel Gherardino il torneo il cui vincitore avrebbe sposato la Fata Bianca è posto in posizione di grande rilievo, alla fine della storia; il Liombruno conduce invece la quête dell'eroe per tutt'altre avventure; ma la circostanza nella quale Liombruno tradisce il suo segreto è proprio in un torneo, che ha per premio la mano di una principessa (ma Liombruno aveva già l'amore della sua donna, dunque che motivo aveva di parteciparvi?), e in cui compare, all'improvviso, come avversario principale, un "sultano" mai menzionato prima: ma che aveva invece la sua ragion d'essere nel Gherardino, dove il Sultano è il tiranno che aveva imprigionato l'eroe; e sua moglie, nel liberare Gherardino per permettergli di partecipare al torneo, lo aveva pregato di ucciderle il marito, divenuto vecchio e disutile. Sembra quindi plausibile che il Liombruno riprendesse il motivo del torneo dal Bel Gherardino. ${ }^{32}$ D'altra parte, la gara di vanti che segue il torneo, a cui dapprima Liombruno non partecipa, ma che determina, dietro le insistenze del re, la rivelazione del segreto, è la stessa indetta (non si capisce bene con quale intenzione di vendetta) dalla regina Ginevra, respinta da Galvano, della Ponzela Gaia: anche qui, dapprima Galvano si ritrae e solo a causa dei rimproveri della regina rivela la sua "bela amanza." Come nel Liombruno, l'apparizione della fata che salva il cavaliere da morte comporta anche i rimproveri della donna all'amante sleale, e coincide con la separazione e l'inizio della quête.

E qui, la storia di Lanval potrebbe esser finita (principalmente perché la mia ricerca non è andata oltre le soglie del 3-400). ${ }^{33}$ 
Come si è visto, il lai di Marie de France, pur essendo solo uno dei 12 lais della raccolta, per la forza della sua poesia, per il prestigio dell'ambiente sociale in cui si è diffuso, per la familiarità dei motivi trattati, ha esercitato tanta suggestione da ispirare ed essere incorporato in parecchie altre opere, anche appartenenti a generi diversi: il lai Graelent, il poema Partonopeus de Blois, la novella cortese La Châtelaine de Vergi; una fusione della storia di Lanval e del Partonopeus de Blois ha dato luogo a una parte del Bel Inconnu. A loro volta, ciascuna di queste opere si pone a capostipite di un'altra serie di imitazioni e di traduzioni. Per seguire soltanto il filone italiano: la Châtelaine de Vergi dà luogo al cantare della Dama del Verzú e, piú tardi, alla novella del Bandello; ${ }^{34}$ Le Bel Inconnu insieme al Partonopeus de Blois e al Graelent danno luogo al Bel Gherardino; ma, intanto, il lai di Lanval nella sua forma originaria si fa conoscere in Italia e il Bel Gherardino con il lai di Lanval (insieme a motivi di provenienza diversa) danno luogo al Liombruno; il Liombruno e il Lanval (in combinazione con altri motivi della tradizione arturiana) danno luogo alla Ponzela Gaia.

\section{Le versioni novellistiche. Boccaccio}

Ma, nonostante quest'intreccio di prestiti all'interno del genere canterino, bisogna ammettere che le rielaborazioni italiane non hanno avuto la forza di generare altre incarnazioni e perpetuare sotto altra forma la storia di Lanval. Nessuna, salvo una. Per la verità, non si tratta della storia di Lanval nel suo complesso, ma soltanto di una porzione di essa sotto la forma che aveva assunto nel cantare del Bel Gherardino, influenzata dalla conoscenza del Partonopeus, e rifusa nell'immaginazione di un grande scrittore: ancora una volta, il Boccaccio. ${ }^{35}$ La novella $3^{\text {a }}$ della $2^{\text {a }}$ giornata (consacrata, come si sa, a "chi, da diverse cose infestato, sia oltre alla sua speranza riuscito a lieto fine") ci presenta il nipote di una famiglia di mercanti, caduti in miseria per spese inconsiderate, il quale, esercitata l'usura per un po' di tempo in Inghilterra e in viaggio per tornare in patria, si accompagna al seguito di un "abate bianco." L'abate, che è in realtà la figlia del re d'Inghilterra sotto mentite spoglie, si innamora del giovane Alessandro; a notte, lo invita nel suo letto, gli si fa conoscere per donna e gli si concede; sposatisi poi ufficialmente davanti al Papa, i giovani risollevano finalmente le sorti della famiglia. Ben poco è rimasto qui, certo, del Lanval; ma sí del Partonopeus: l'incontro nel letto, di notte, la regalità (di un regno tutto umano) della fanciulla; ma sí del Gherardino: la povertà e la nobiltà d'animo del giovane, il con- 
testo borghese, il Papa, la "bianchezza" della donna, la gioiosa rapidità della soluzione amorosa. Insomma, quello che rimane della storia di Lanval è la primissima parte, la vicenda di un giovane nobile che acquista, insperatamente, l'amore di una donna ricchissima, beneficiando della sua generosità; l'amore è, naturalmente, inteso in senso completo; fra Lanval e Alessandro sta l'innovazione fondamentale derivata dalla favola di Amore e Psiche e introdotta dal Partonopeus.

È significativo che la novella boccacciana sia il racconto che, piú di ogni altro, spinge a fondo la rielaborazione della storia, fin quasi a renderla irriconoscibile. È una prova di piú della diversità radicale del genere novella (particolarmente nella sua accezione boccacciana) da tutti quelli che hanno fornito al Boccaccio trame e motivi. Sparita ogni traccia di meraviglioso, il Boccaccio immette la vicenda in un mondo mercantile, contemporaneo e quotidiano ("Camminando adunque il novello abate ora avanti e ora appresso alla sua famiglia, sí come noi tutto il giorno veggiamo per cammino avvenir de' signori"). Persino l'alone di fasto esotico, che rimane nell'umanizzazione della fata del Partonopeus come principessa dell'impero favoloso di Bisanzio, è qui volutamente ridotto al minimo, nascosto e soffocato com'è dal travestimento della donna e dalla trasformazione della sua corte in una carovana di frati al servizio di un abate. La bontà e la nobiltà del protagonista, nei lais e nei poemi è un presupposto, un dato indiscusso e la causa implicita dell'avventura che gli capita. Nella novella invece viene dato rilievo piú alle circostanze che portano il protagonista in una data situazione, che al carattere dell'eroe; le azioni e reazioni di quest'ultimo sono spiegate, in una luce tutta umana, dalle giustificazioni psicologiche che il Boccaccio trova per ogni gesto dei suoi personaggi; ed ecco l'insistenza del Boccaccio sull'antefatto, sulle vicende della famiglia di Alessandro; addirittura, la nobiltà del giovane è piú nelle considerazioni della principessa che si è innamorata di lui che un dato di fatto. La novella è il resoconto di un fatto straordinario, ma avvenuto in un mondo a cui narratore e ascoltatori appartengono tanto quanto i protagonisti della novella; avvenuto secondo modalità assolutamente giustificate e giustificabili agli occhi dei lettori del Decameron. ${ }^{36}$ E chiaro allora che una avventura straordinaria è sufficiente a costituire un racconto. Manca perciò nella novella la caratteristica sequenza che vede il protagonista perdere il bene acquistato per sua colpa, il pentimento, l'espiazione, la quête e il finale riacquisto della felicità, che è tipica della storia di Lanval. Strutturalmente troviamo invece 
nella novella una costruzione ascendente, culminante con il matrimonio davanti al Papa, seguito dalla breve fase discendente dell'epilogo che, dando rapidamente uno scorcio di quella che è stata la vita dei protagonisti dopo quel fatto straordinario, fa rientrare la "novella" nella storia, al livello normale dell'esistenza umana e civile.

\section{Ser Giovanni Fiorentino. Giovanni Sercambi ${ }^{37}$}

La novella boccacciana ha dato luogo a due rielaborazioni novellistiche, la $3^{a}$ della prima giornata del Pecorone del fiorentino Ser Giovanni, e l'LXXXV del Novelliere del lucchese Giovanni Sercambi.

I dati che hanno piú colpito l'immaginazione dei rielaboratori sono il travestimento ecclesiastico (nel Pecorone una nobile dama di Viterbo è vestita da frate, nel Novelliere la figlia del re di Spagna è parata come un vescovo), l'accompagnamento in viaggio e, naturalmente, il motivo erotico dell'uomo che, condotto per ragioni varie nel letto del falso ecclesiastico, scopre che il compagno è femmina. Delle due rielaborazioni, quella del Sercambi si tiene stretta alla fonte boccaccesca, conservando la professione mercantile del protagonista maschile, la storia della sua famiglia, la natura regale della donna; la novella del Pecorone, invece, colloca la storia all'interno di un mondo esclusivamente ecclesiastico (in conformità, a quanto pare, con la natura monacale dei protagonisti della cornice): prete il protagonista maschile, prete travestito da frate l'accompagnatore del falso frate, amante di un cardinale la donna, cardinale il personaggio presso il quale termina il viaggio. La storia non si conclude con un matrimonio ma con una separazione: risistemata la donna presso il cardinale e ottenuti da lui tutti i favori che poteva desiderare, per l'intercessione della donna, il protagonista maschile non può che tornare in patria:

... di che parve molto duro alla Petruccia, e pure, veggendo la volontà sua, fu contenta. . . La Petruccia . . . si cavò di dito uno bellissimo anello e donògliele e disse: "Tè, porta questo per mio amore e nol donare mai a nessuna che non sia piú bella di me." (Pp. 76-77)

A questo punto si ricorderà che apparteneva alla tradizione della storia, a partire dal Partonopeus, al Bel Inconnu, al Bel Gherardino, al Liombruno, il motivo del commiato dell'eroe dalla fata per tornare in patria; particolarmente vicini i cantari italiani: 
"Berie che il mio core del tuo partire tormenta po' ch'a 'tte piace, ed io ne sono contenta." (Bel Gher., I, 34, 7-8)

Ed ella allora gli donò un anello che da disagio scampasse il donzello. (Liombruno, 175-76)

C'è persino, nelle parole della Petruccia, un'eco della proibizione della fata di rivelare la sua esistenza. Ancora una volta, cioè (come nel caso del Graelent), un continuatore ha reinserito la sua fonte principale in un contesto di storie consimili, attingendo liberamente dalla tradizione di quella storia, e contaminandone i motivi con quelli della sua fonte principale. ${ }^{38}$

\section{Conclusione}

Arrivati alle soglie del '400, abbiamo dunque rintracciato la continuità di una storia, viva per tre secoli, e abbiamo cercato di osservare le modifiche a cui la trama di base va incontro nell'incorporarsi in generi diversi da quello in cui la storia fu fusa originariamente. Nello stesso tempo, abbiamo cercato di gettar luce su come funzionava l'immaginazione di uno scrittore medievale che prendeva a nucleo della sua creazione una storia precedente. La nozione di fonte viene in questo modo illuminata di nuova luce, apparendo chiaro che l'autore medievale, nel momento in cui sceglie una particolare articolazione di avvenimenti derivata da un'opera precisa, tende però a tener presente tutta la tradizione di quella storia, ed è alle storie appartenenti al medesimo filone immaginativo (spesso piú antiche della "fonte") che ricorrerà per prima cosa nell'introdurre le sue varianti. Il riconoscimento di questo meccanismo, mentre permette di non moltiplicare indebitamente le entità perdute, ci costringe anche ad ammettere la persistenza di forme antiche, particolarmente se sorrette dal prestigio della loro codificazione letteraria e dell'ambiente sociale a cui appartenevano. Tali forme antiche interferiscono spesso e volentieri con storie da loro derivate e concorrono alla creazione di ulteriori rielaborazioni. Estremamente innovatore, anche in questo caso, il Boccaccio, che frantuma e rielabora in una forma tutta sua i dati della tradizione, appena riconoscibili sotto la novità della forma boccacciana.

Tuttavia, queste vicende non saranno da considerarsi come fenomeni necessari della narrativa in quanto tale, ma saranno da vedere sempre in collegamento con le concrete condizioni storiche nelle quali tali vicende si verificano. È molto probabile che la nuova ondata di temi (di opere) di origine francese nell'Italia della 
metà del Trecento (si ricordi che per quanto riguarda la materia epica e la letteratura franco-veneta il Rajna considerava chiusa con la metà del '200 la trasmissione di opere dalla Francia all'Italia; e per l'influsso della poesia trovadorica la cronologia non è molto diversa) sia da mettere in relazione con il consolidarsi nell'Italia meridionale di una dinastia francese, con cui i fiorentini intrattengono importanti rapporti. Allo stesso tempo, l'apparizione scritta di opere "giullaresche" in manoscritti mercantili andrà forse ricollegata ad un nuovo status che l'antico giullare raggiunge proprio in questo periodo: è infatti intorno alla metà del Trecento che compare la figura del canterino ufficialmente assunto, per esempio, dai Comuni di Perugia e di Firenze, con il compito di allietare i Priori ma anche, nel suo tempo libero, i buoni cittadini del Comune. È plausibile che questi canterini, innalzati ad una carica stabile nell'ambiente del piú alto potere comunale, se anche non possono gareggiare, naturalmente, con la nuova classe colta che dà alla letteratura italiana le sue realizzazioni piú notevoli, cercassero di mantenersi all'altezza del loro impiego rinnovando il loro repertorio e rivolgendosi a quella materia cavalleresco-cortese che tanto favore aveva ottenuto nelle corti francesi, e che la presenza della corte angioina in Italia aveva reso di nuovo facilmente accessibile.

Il vario intreccio dei motivi, delle forme, dei generi, persino quando alla base di una serie di entità letterarie è riconoscibile una sola fabula, e i collegamenti con la storia che tali vicende ci permettono di intravedere sono i motivi per cui rimane ancora cosí difficile lo studio dei generi narrativi minori come i cantari e le novelle non boccacciane; ma è anche, d'altra parte, ciò che lo rende affascinante.

\section{Université de Montréal}

\section{NOTE}

1 T.P. Cross, 10.

2 Parte del Book of Leinster, datato intorno al 1150. Traduzione inglese in Ancient Irish Tales, pp. 208-10. Il mio riassunto è desunto dall'articolo di T.P. Cross.

3 Segnalata come appartenente al "ciclo della scommessa" da G. Paris, "Le cycle de la gageure" in Romania XXXII (1903), 483. Tradotto recentemente da P.K. Ford in The Mabinogi and other Medieval Welsh Tales, pp. 164-81. La Storia di Taliesin ci è stata conservata solo in un documento del XVI ${ }^{\circ}$ secolo, una raccolta del patrimonio narrativo gallese ad opera dell'erudito Elis Gryffid. Gli studiosi di mitologia celtica ritengono però che il materiale cosí conservato risalga a rispettabile antichità.

4 Per rendersi conto della possibilità di una comparazione cosí strana fra una donna e dei cavalli, bisognerà rifarsi alla mentalità della primitiva società celtica 
in cui "equinità" e "femminilità" erano entrambi attributi della sovranità: cfr. P.K. Ford, "Introduzione," p. 9.

5 The Mabinogi and other Welsh Tales, pp. 167-69.

6 "En définitive, le lai est un petit conte en vers qui développe avec sobriété une intrigue romanesque dont la narration même (les médiévaux diraient: la conjointure) prévaut sur le fond ou sens, et qui cultive volontiers l'émotion contenue à travers un langage assez délicat." (P. 47).

7 Payen precisa ancora: "Ce schéma pourrait s'appliquer à beaucoup d'oeuvres narratives médiévales; mais le lai a ceci de particulier qu'il relate une quête du bonheur considéré comme une fin en soi; or, la quête qui fait l'objet du roman, par exemple, chez Chrétien de Troyes, est d'une autre nature, puisqu'elle vise une plénitude fondée sur une prouesse généreuse: le bonheur s'y atteint, mais comme par surcroît. Le lai, lui, ne s'oriente que par rapport à la chasse au bonheur: une fois de plus, il apparaît que structure et sens sont indissociables." (P. 49).

8 Cfr. C. Payen, p. 41.

9 In questo articolo limitiamo lo studio al lai de Graelent come il piú interessante e il piú vicino alla storia del Lanzal. Ma altri lais anonimi rivelano chiaramente una discendenza dal lai di Maria, in particolare Guingamor e Désiré. Ugualmente per quanto riguarda i poemi limitiamo lo studio al Partonopeus e al Biaus Desconeüs, senza ignorare che altri poemi hanno imitato motivi caratteristici del Lanval: in particolare, la scena finale del Guillaume de Dole, di Jean Renart, in cui Lienor si reca al cour plenière, convocata dall'imperatore Corrado, per confondere l'impostura del siniscalco è identica al finale di Lanval.

Del resto, i lais di Maria sembrano essere stati una fonte d'ispirazione privilegiata per gli autori di poemetti cortesi. Cosí, il lai du Fraisne è confluito, insieme ad altre fonti, nel Galeran de Bretagne e nell'Escoufle, l'Eliduc ha dato luogo all'Ille et Galeran (cfr. Hoepffner 1930).

10 Esistono due versioni inglesi del Lanval: una che si tiene stretta al testo di Marie de France, pubblicata dal Kittredge, l'altra, dal titolo Launfal Miles è opera di Thomas Chestre, che combina Lanval e Graelent e introduce alcuni elementi spuri.

11 Nella versione del Tristano di Gottfried di Strasburgo, vv. 3584-85, nell'Erec et Enide di Chrétien de Troyes, v. 1952. Nella Chanson d'Aspremont Graelent è menzionato come l'autore del primo lai bretone.

12 A. Fourrier restringe il periodo d'attribuzione agli anni 1182-85: cfr. p. 384.

13 Cfr. "Introduzione" di L. Smith, vol Il, parte 2a, pp. 5-13.

14 Difficile del resto determinare se il Désiré attinge al Partonopeus o viceversa, data la datazione incerta dei lais anonimi.

15 Anche se le Metamorfosi di Apuleio rimasero sconosciute fino al XIll ${ }^{\circ}$ secolo, l'autore del Partonopeus poteva aver trovato la favola di Amore e Psiche nelle Mythologiae di Fulgenzio che furono invece un testo noto e utilizzato: cfr. A. Fourrier, pp. 385-86.

16 Cfr. J.L. Weston. Ma, secondo il Fourrier (pp. 447-48), è l'Ipomedon a imitare il Partonopeus.

17 "Toute quête, dans le roman, est fondamentalement eschatologique. La découverte et la conquête de l'objet rechercé mènent à chef une action précédemment entreprise; elles n'épuisent pas la destiné du héros: les virtualités de l'agent. La fin de la quête, la fin même du roman (si elles coïncident) représentent un palier, une étape. Le temps continue au de là." (P. 357).

18 ". . . du seul fait qu'une succession et un ordre irréversible sont imposés aux diverses parties de l'exposé registral . . . le déroulement du temps est à peine sensible; la conclusion est virtuellement donnée dès le début." (P. 381).

19 Cfr. Fourrier, pp. 448-49.

20 Ancora dibattuti i rapporti fra il testo francese del Bel Inconnu e la redazione inglese (XIV $\mathrm{XVc}^{\mathrm{O}}$.) del Lybeaus Desconus, in cui manca il motivo finale del torneo. 
Per le ulteriori derivazioni del Bel Inconnu e il motivo del Fair Unknown nella letteratura medievale cfr. M. Mills, "Introduzione," pp. 42-50.

21 R. Dubuis, p. 525.

22 Per le edizioni cfr. A. Moret, pp. 22-26; imitazioni del Partonopeus sono state riconosciute, oltre che nel Bel Inconnu, nel Florimont di Aimon de Varennes (Fourrier, pp. 449 ss.) e nel poema tedesco Frédéric de Souabe (Moret, p. 101).

23 Cfr. E. Levi, pp. 26-36; A. Moret, pp. 103-04.

24 A. Fourrier, pp. 368-69.

25 Il motivo potrebbe avere la sua lontana origine della Vita Merlini di Geoffrey de Montmouth, in cui Merlino, per il dolore di aver perduto tre fratelli in battaglia, si riduce a nutrirsi solo di frutta e riempie la foresta di Caledonia dei suoi lamenti, fatto silvester homo. Prima di arrivare al Partonopeus, potrebbe esser passato per l'Yvain di Chrétien, oltre che per i lais collegati alla storia di Lanval.

26 E. Levi, p. 35. Al Graelent dovranno essere attribuiti anche, allo stadio attuale delle conoscenze, il motivo della povertà del cavaliere, il tabú consistente nel silenzio e la generosità del cavaliere, fornito all'improvviso di una ricchezza di cui non si conosce l'origine, poiché né il Partonopeus né il Bel Inconnu conservano questi motivi.

27 E il duello si conclude con un gran colpo di spada sulla testa dell'avversario:

E Gherardin si levò prestamente colla spada tagliente sanza fa resta, e inver dell'orso nequitosamente un colpo che gli dè sopra la testa, che l'ebbe fesso infino al bianco dente; e Marco Bello ne facea grande festa.

(Bel Gherardino, I, 17, 1-6)
Puis le refiert en la cervele,

Li chevaliers tos en chancele;

La coiffe del hauberc trencha,

Desi qu'al test li fers ala;

Par la cervele met l'espee,

Dusques es dens li est colee.

Les gens crïent et joie ont grant.

(Bel Inconnu, vv. 2181-87)

28 Cosí la fata di Lanval gli era apparsa "en sa chemise senglement" (v. 99):

Tut ot descovert le costeé

Le vis, le col e la peitrine.

Plus est blanche que flur d'espine. (Vv. 104-06)

29 Curioso che già nella traduzione del Tristano di Gottfried di Strasburg si dia lo stesso appellativo a Graelent: "et ce fu le lai de la fière amie du beau Graelent," vv. 3584-85. (J. Bedier, Le roman de Tristan par Thomas, vol. I [Paris: Didot, 1902] p. 52).

30 Cfr. M. Bendinelli-Predelli, "Dal cantare romanzesco al cantare novellistico: vicissitudini di una forma" in Genèse, rayonnement ecc., pp. 174-88.

31 Come già notava V. Branca, "Nostalgie tardogotiche e gusto del fiabesco nella tradizione narrativa dei cantari" in Studi di varia umanità in onore di F. Flora (Milano: Mondadori, 1963), pp. 88-108.

32 Sono già state rilevate piú volte vicinanze di fraseggio fra il Liombruno e il Bel Gherardino. Alle note, vorrei aggiungere questa, interessante perché si riferisce non alla versione vulgata del Liombruno ma al frammento pubblicato dal Varanini, che rappresenta forse una versione piú antica della vulgata: la Fata che entra nel letto del Bel Gherardino gli dice subito, per rassicurarlo:

"Non avere di me pavento,

ch'io sono colei che 'tti farò contento" (I, 23, 7-8);

e l'aquila, venendo a prendere il fanciullo Liombruno rimasto solo su un'isoletta,

... disse: "Levate su dritto,

[io son] colei che te farrò contento" (vv. 85-86);

dove inoltre contento del v. 86 rima con pavento del v. 84 . 
33 Il frammento pubblicato da Varanini in appendice al cantare della Ponzela Gaia si riferisce alla parte finale del cantare, che non ha riscontri precisi con la storia di Lanval; gli accenni alla Gaia Donzella che troviamo nella Tavola Rotonda (cfr. Levi, pp. 43-45) sono troppo vaghi per poter riconoscere la storia a cui il romanziere alludeva. Per quanto riguarda il Liombruno, è nota la lunga fortuna che la novella ha riscosso fino al secolo scorso: cfr. l'elenco delle edizioni a stampa fornito dal Varanini in Sul testo, sull'attribuzione ecc.

34 Per i rapporti fra le varie redazioni cfr. lo studio di Franceschetti.

35 Cfr. M. Bendinelli-Predelli, "Del Boccaccio e del Bel Gherardino" in Studi sul Boccaccio XIII (1982), 363-79.

36 "Au lieu du général, la nouvelle souligne le particulier, le cas type est remplacé par le cas d'espèce caractérisé par des circonstances particulières. La règle, le principe et la loi cèdent la place à l'irrégulier, à l'exception, à l'événement inoui. Le destin et la providence sont relayés par le hasard et la fortune. La nécessité est supplantée par la liberté: c'est-à-dire les protagonistes ne sont plus les simples représentants d'un sens préétabli et incontestable, mais ils doivent s'affirmer par eux-mêmes et jouissent d'une autonomie insoupçonnée jusqu'alors. Au lieu d'une solution ou d'une véritable conclusion, nous rencontrons souvent dans la nouvelle un résidu insoluble ou une fin en suspens. Et enfin, la nouvelle ne confirme pas par principe un ordre des choses ou une norme, au contraire elle les remet précisément en question dans nombre de cas ..." H.-J. Neuschäfer, p. 109.

37 Il motivo della principessa travestita da vescovo (versione del Sercambi) sarà ripresa nel Cinquecento da Francesco Sansovino nel Centonovelle scelte, I, 5.

38 Un'eco del Lanval e del Partonopeus sembra potersi avvertire anche nella novella $139^{a}$ del Sercambi quando il giovane principe, ridotto allo stato di umile servitore dallo zio, incontra la principessa Biancamontagna sotto una tenda: "E quando il Nibbio fu al messo della piassa, e vidde uno padiglione con un lume, dentro entrò: vidde un bellissimo letto, in nel quale vide una bellissima giovana la quale ancora non dormia. . . ." La principessa farà poi ricchi doni al Nibbio, il quale parteciperà in incognito al torneo di tre giorni e, riuscito vincitore, sposerà naturalmente la principessa.

\section{EDIZIONI}

Testi celtici:

Aidead Muirchertaig maic Erca, a c. di Whitley Stokes in Revue Celtique XXIII (1902), 396 ss.

Togail Bruidne Dá Derga, pubblicato e tradotto da Whitley Stokes in Revue Celtique XXII (1901), 14 ss.

The Book of Leinster, formerly Lebar na Núachongbála, a c. di R.I. Best, O. Bergin et M.A. O'Brien. Dublin: Institute for Advanced Studies, 1954.

The Mabinogi and other Medieval Welsh Tales, tradotte da P.K. Ford. Berkeley-Los Angeles-London: University of California Press, 1977.

Ancient Irish Tales, a c. di T.P. Cross e C. Slover, con bibliografia a c. di W. Dunn. New York: Barnes \& Noble, 1969.

\section{Testi francesi}

Marie de France, Lais, a c. di J. Lods. Paris: Champion, 1959.

P.M. O'Hara Tobin, Les lays anonymes des XII et XIII siècles. Genève: Librairie Droz, 1976. 
Partonopeus de Blois, a c. di G.A. Crapelet, "Analyse critique" di A.C.M. Robert. Paris: Crapelet, 1834, voll. 2.

Partonopeu de Blois, a c. di J. Gildea, "Introduzione" di L. Smith. Villanova (Penn.): Villanova University Press, 1970, voll. 2.

Renaut de Beaujeu, Le Bel Inconnu, a c. di G.P. Williams. Paris: Champion, 1929.

Floire et Blanchefleur, a c. di M. Pelan. Paris: Les Belles Lettres, 1937.

R.E.V. Stuip, La Chastelaine de Vergi, édition critique du ms. BN fr 375 , avec Introduction, Notes, Glossaire et Indes, suivie de l'édition diplomatique de tous les mss. connus du XIII et XIV siècle. The Hague-Paris: Mouton, 1970.

Testi inglesi:

G.L. Kittredge, "Launfal (Rawlison Version)" in American Journal of Philology X (1889), 1-33.

Launfal Miles in Middle English Metrical Romances, a c. di W.H. French e C.B. Hale. New York: Prentice Hall, 1930, pp. 345-80.

Lybeaus Desconus, a c. di M. Mills, "Early English Text Society." Oxford: Oxford University Press, 1969.

Testi italiani:

G. Boccaccio, Teseida, a c. di A. Limentani, in Tutte le Opere, vol. II. Milano: Mondadori, 1964.

Il Libro di Fiorio e Biancifiore e Cantare del Bel Gherardino in D. de Robertis, "Cantari antichi," Studi di Filologia Italiana XXVIII (1970), 80-133.

Liombruno in Poeti minori del Trecento, a c. di N. Sapegno. Milano-Napoli: Ricciardi, 1952. Pp. 843-68.

Un frammento rappresentante una diversa redazione pubblicato da G. Varanini in Sul testo, sull'attribuzione ecc..

G. Varanini, Ponzela Gaia. Bologna: Commissione per i testi di lingua, 1957.

P. Rajna, I cantari di Carduino, giuntovi quello di Tristano e Lancillotto quando combattettero al petrone di Merlino. Bologna: Commissione per $\mathrm{i}$ testi di lingua, 1968 ( $1^{\text {a }}$ ediz.: 1873).

Ser Giovanni, Il Pecorone, a c. di E. Esposito. Ravenna: Longo, 1974.

G. Sercambi, Il Novelliere, a c. di L. Rossi. Roma: Salerno ed., 1974. Voll. 3.

Studi:

AA.VV., Genèse, condification et rayonnement d'un genre médiéval: la nouvelle, a c. di M. Picone, G. di Stefano e P. Stewart. Montreal: Plato Academic Press, 1983.

AA.VV., I Cantari. Struttura e tradizione, a c. di M. Picone e M. BendinelliPredelli. Firenze: Olschki, 1984.

T.P. Cross, "The Celtic Elements in the Lays of Lanzal and Graelent," in Modern Philology, XII (April 1915), 1-60.

R. Dubuis, Les "Cent Nouvelles nouvelles" et la tradition de la nouvelle en France au Moyen Âge. Grenoble: Presses Universitaires de Grenoble, 1973.

E. Esposito, "Introduzione" a Il Pecorone. Ravenna: Longo, 1974. Pp. VIIXXXVI. 
P.K. Ford, "Introduzione" a I Mabinogi and other Welsh Tales. Berkeley- Los Angeles-London: University of California Press, 1977. Pp. 1-31.

L. Foulet, "Marie de France et les lais bretons" in Zeitschrift für Romanische Philologie, XXIX (1905), 19-56.

A. Fourrier, Le courant réaliste dans le roman courtois en France au Moyen Âge. Paris: Nizet, 1960.

A. Franceschetti, "Dal cantare alla novella: La donna del Vergiú e Matteo Bandello," in AA.VV., I Cantari. Struttura e tradizione, pp. 161-76.

J. Frappier, "Remarques sur la structure du lai. Essai de définition et de classement" in La littérature narrative d'imagination. Des genres littéraires aux techniques d'expression. Paris: Presses universitaires de France, 1961. Pp. 23-39.

E. Hoepffner, "Les lais de Marie de France dans Galeran de Bretagne et Guillaume de Dole," in Romania LVI (1930), 212-35.

E. Hoepffner, Les lais de Marie de France. Paris: Nizet, 1959.

P. Lakits, La "Châtelaine de Vergi" et l'évolution de la nouvelle courtoise. Debrecen: Kossuth Lajos Tudományegyetem, 1966.

E. Levi, I cantari leggendari del popolo italiano nei secc. XIV e XV, Suppl. al Giornale storico della letteratura italiana n. 16, Torino, 1916. M. Mills, "Introduzione" a Lybeaus Desconus, EETS, Oxford University Press, 1969.

A. Moret, L'originalité de Conrad de Wurzbourg dans son poème "Partonopier und Meliur." Lille: Société de l'édition du Nord, 1933.

H.-J. Neuschäfer, "Boccace et l'origine de la nouvelle. Le problème de la codification d'un genre médiéval," in AA.VV., Genèse, codification ecc., pp. 103-10.

H. Newstead, "The Background of Partonopeus de Blois," in Publications of the Modern Language Association, LXI (1946), 916-46.

C. Payen, Le lai narratif, "Typologie des sources du Moyen Âge occidental," fasc. 13. Brepols: Turnhout, 1975.

L. Rossi, "Introduzione" a G. Sercambi, Il Novelliere. Roma: Salerno ed., 1974. Pp. IX-LXI.

W.H. Schofield, "The Lays of Graelent and Lanzal and the Story of Wayland," in Publications of the Modern Language Association, XV (1900), 121-80.

C. Segre, "Lanval, Graelent, Guingamor," in Studi in onore di Angelo Monteverdi. Modena: Società Tip. Editrice Modenese, 1959. Pp. 756-70.

G. Varanini, "Sul testo, sull'attribuzione e su una inedita redazione della Storia di Liombruno" in Studi mediolatini e volgari II (1954). Pp. 251-81.

G. Varanini, "Introduzione"t alla Ponzela Gaia. Bologna: Commissione per i testi di lingua, 1957.

H. Weinrich, "Structures narratives du mythe" in Poétique I (1970).

J.L. Weston, The Three Days Tournament. London: David Nutt, 1902.

P. Zumthor, Essai de poétique médiévale. Paris: Seuil, 1972. 